\title{
3 Die Wiederentdeckung Herkulaneums, der Villa und der Papyrusrollen
}

Es ist viel Unheil in der Welt geschehen, aber wenig, das den Nachkommen so viel Freude gemacht hätte. Ich weiß nicht leicht etwas Interessanteres.

Goethe über die vom Vesuv verschütteten Städte $(1787)^{21}$

\subsection{Die Entdeckung Herkulaneums als Urmoment des Klassizismus}

Nach einem über anderthalb Jahrtausende währenden Dornröschenschlaf wurden im Bereich des antiken Herkulaneum um 1710 bei Brunnenbauarbeiten zufällig Marmorstatuen gefunden, was Grabungen, heute würde man sagen Raubgrabungen, auf Geheiß von Emanuel-Maurice von Lothringen, Prinz d' Elboeuf, anstieß. Tunnel wurden in das Gelände hineingetrieben und man suchte gezielt nach Kunstschätzen. Die meisten Objekte fand man im Umkreis des für eine solche Kleinstadt doch sehr respektablen Theaters von Herkulaneum, welches sich in den folgenden Jahrzehnten zu einem regelrechten touristischem Magneten entwickelte und als Höhepunkt der unterirdischen Stadt galt. ${ }^{22}$ Hier wurden etwa die berühmten „Herkulanerinnen“ gefunden, welche durch einige Umwege nach Dresden an den Hof von August III. gelangten. ${ }^{23}$ Jedoch waren es erst die Bourbonischen Grabungen zwischen 1738 und 1780, welche in großem Stile die Kunstschätze Herkulaneums zu Tage förderten. Diese entfalteten einen kaum zu überschätzenden Einfluss auf die Entwicklung des Klassizismus und das Geistesleben in Europa. Erst im Laufe des 18. Jahrhunderts begann Pompeji, im Hinblick auf Ruhm und Popularität Herkulaneum den Rang

21 Johann Wolfgang von Goethe, Italienische Reise, 2. Teil, Neapel, 13. März 1787.

22 Noch heute liegt das Theater unterirdisch, kann aber $\mathrm{zu}$ gewissen Zeiten (wieder) von Touristen besucht werden.

23 Vgl. Maria Guidobaldi, ,Schatzgräber und Archäologen. Die Geschichte der Ausgrabungen von Herculaneum,“ in Verschüttet vom Vesuv. Die letzten Stunden von Herculaneum (hg. von Josef Mühlenbrock und Dieter Richter; Mainz, 2006), (17-26). Longo Auricchio et al., La villa (wie Anm. 3), 21-36. Mario Capasso, „Carlo di Borbone e la papirologia ercolanese“, in Ianua Classicorum: temas y formas del mundo clásico Bd. 3 (hg. von Jesús de la Villa Polo/Patricia Cañizares Ferriz/Emma Falque Rey/José Francisco González Castro/Jaime Siles; Madrid, 2016), (35-41). Die Tochter von August III, Maria Amalia Christina, war mit dem König Neapels Karl III. von Bourbon verheiratet. Für ihre Beziehung zu den Herkulanischen Papyri siehe Giuliana Leone, „Maria Amalia di Sassonia e i papiri ercolanesi,“ CErc 49 (2019): (163-172). 
abzulaufen, als dort großflächig immer mehr Ausgrabungen unter freiem Himmel zu bestaunen waren. In Herkulaneum dagegen wurden fast sämtliche Funde in später meist wieder zugeschütteten Stollen gemacht und an der Oberfläche, wo der Ort Resina über dem antiken Herkulaneum lag, waren kaum Antiquitäten für Besucher sichtbar. Die Kunstgegenstände wurden zunächst nach Portici (nahe Herkulaneum) in ein dem Königspalast angegliedertes Museum verbracht und kamen erst zu Beginn des 19. Jh. nach Neapel (in das heutige Museo Archeologico Nazionale di Napoli).

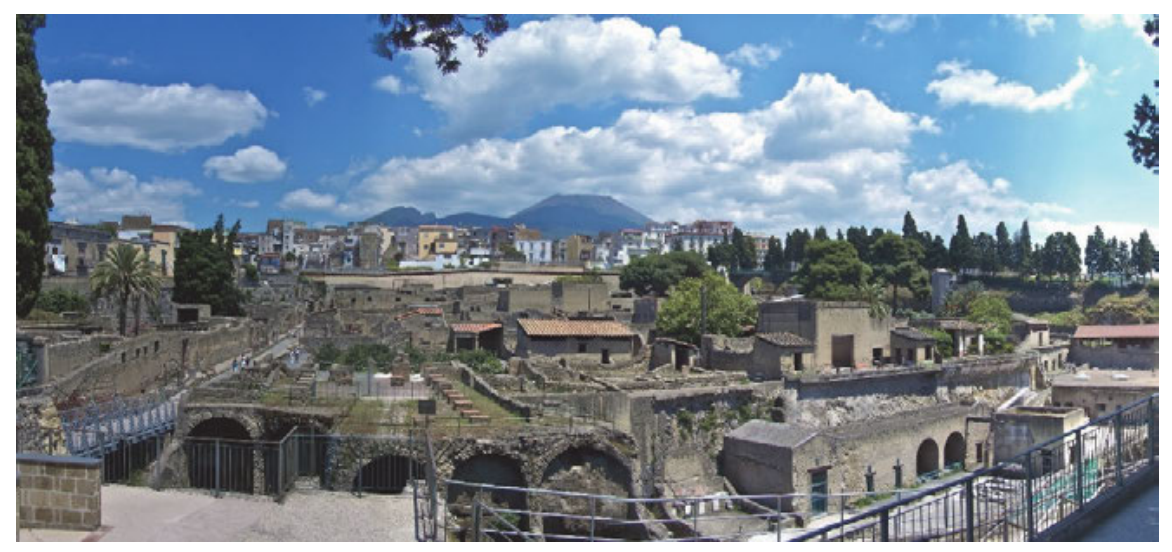

Abb. 5: Die Ausgrabungen von Herkulaneum mit dem Vesuv im Hintergrund

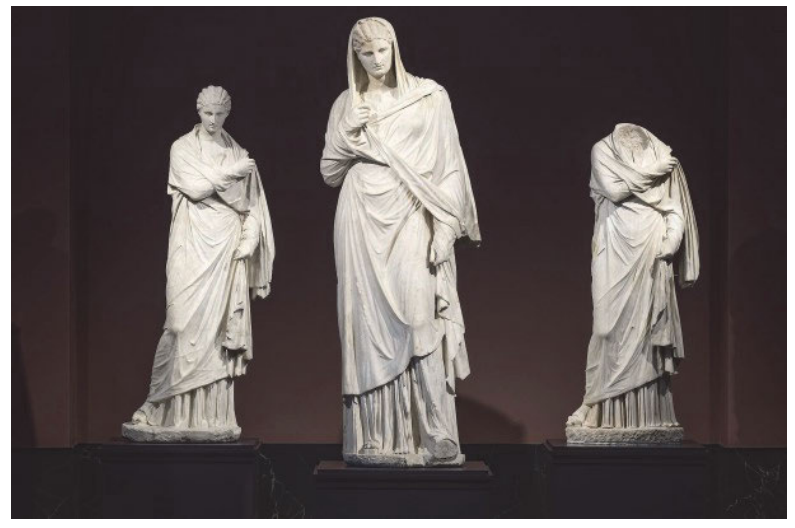

Abb. 6: Herkulanerinnen

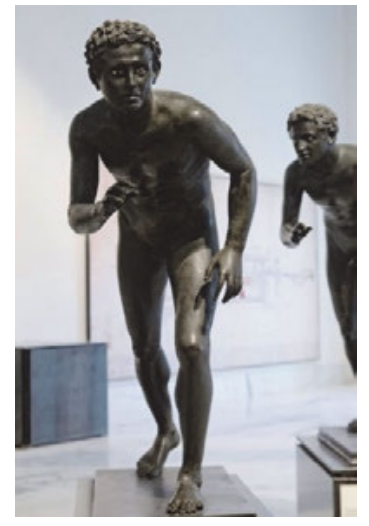

Abb. 7: Läufer aus Herkulaneum 
Kein anderer als Johann Joachim Winckelmann berichtete in seinem „Sendschreiben von den Herkulanischen Entdeckungen“ an den Reichsgrafen von Brühl im Jahre 1762 über die Funde, einschließlich der Papyri, und begründete damit letztlich die Archäologie und Kunstgeschichte. ${ }^{24}$ Der Enthusiasmus des gebildeten Europa über die neuesten Nachrichten von den antiken Stätten am Golf von Neapel kannte im wahrsten Wortsinne keine Grenzen und die Skulpturen sowie Wandmalereien inspirierten alsbald das Interieur zahlreicher adeliger und königlicher Residenzen. Goethes Besuch von Pompeji und Herkulaneum im Jahre 1787 war für all diejenigen, welche auf ihrer „Grand Tour“ durch Italien kamen, gleichsam obligatorisch - bereits sein Vater hatte 1740 Herkulaneum besucht. ${ }^{25}$ Der Vesuv, Herkulaneum und das schon zu Goethes Zeiten greifbarere Pompeji wurden zu idealisierten Sehnsuchtsobjekten und Symbolorten einer von Antikenverehrung geprägten Epoche, deren Ausläufer bis weit in das 20. Jahrhundert hineinreichten. Die Stätten am Golf von Neapel erfuhren musikalische, architektonische, literarische und künstlerische Manifestationen oder Rezeptionen. ${ }^{26}$ Ohne die Entdeckung Herkulaneums und Pompejis wäre die Entwicklung des Klassizismus und der europäischen Geistesgeschichte vermutlich in Teilen anders verlaufen.

24 Johann Winckelmann, Sendschreiben von den Herculanischen Entdeckungen: an den Hochgebohrnen Herrn, Herrn Heinrich Reichsgrafen von Brühl (Dresden, 1762).

25 Johann Caspar Goethe schrieb 1740 über seine Eindrücke im Theater von Herkulaneum: „Wir mussten uns zunächst ausweisen, wer wir waren, und wurden anschließend von einer Wache begleitet...man kann jetzt auch schon die Mauern erkennen, die im heidnischen und phantastischen Geschmack in den beiden Farben Aschgrau und Rot auf ungeschickte Weise bemalt sind. Diese Figuren stellen Götzen und scheußliche, abstoßende Gestalten dar...“ (nach Albert Meier, Johann Caspar Goethe, Reise durch Italien im Jahre 1740 [Übersetzung und Kommentar] [München, 1982], 203-205).

26 Für einen Überblick siehe Dieter Richter, „Herculaneum im Norden. Die Ausgrabungen als europäisches Ereignis, “ in Mühlenbrock und Richter (Hgg.), Verschüttet vom Vesuv (wie Anm. 23), (183-196) und Dieter Richter, „Der ,Brennende Berg‘. Kulturgeschichte des Vesuvs,“ in Mühlenbrock und Richter (Hgg.), Verschüttet vom Vesuv (wie Anm. 23), (223-238). Félicien David komponierte sogar eine Oper „Herculanum,“ welche 1859 uraufgeführt wurde. 2015 wurde die Oper erneut aufgeführt bzw. gespielt (Flemish Radio Choir/Brussels Philharmonic) und ist nun mit ansprechendem Begleitmaterial und Libretto auf CD erhältlich: Félicien David, Herculaneum. Collection: Opéra francais (San Lorenzo de El Escorial, 2015). Zur Einordnung des Werkes siehe Eric Moormann, „Félicien David's Image of Herculaneum,“ CErc 50 (2020): (273284). 

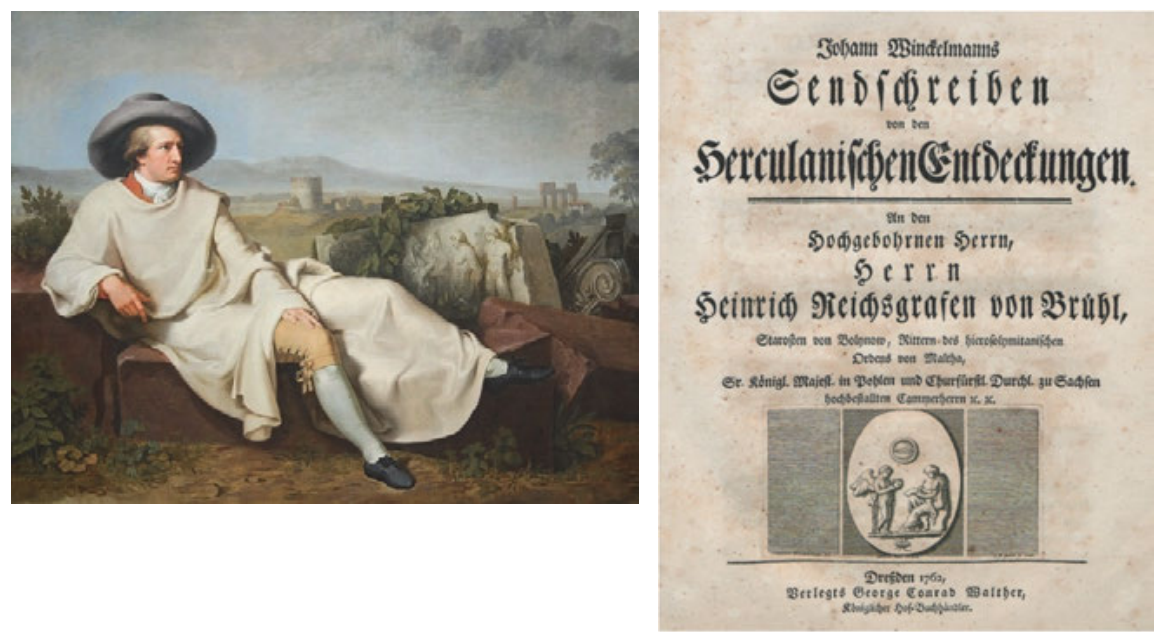

Abb. 8: Der „Dichterfürst“ vor antiker Kulisse

Abb. 9: Winckelmanns Sendschreiben (1762)

\subsection{Die Entdeckung der Villa dei papiri als Urmoment der Archäologie und Kunstgeschichte}

Die Ausgrabungen in Herkulaneum leitete ab 1738 der Pionier-Hauptmann Rocco Gioacchino d'Alcubierre. Im Jahre 1750 entdeckte man die bisher größte bekannte Villa der Antike, die sogenannte Villa dei papiri, seltener auch Villa dei Pisoni genannt. Vermutlich ließ Cäsars Schwiegervater Lucius Calpurnius Piso Caesoninus diese luxuriöse Villa von beeindruckenden Dimensionen als eine Art Sommerresidenz erbauen. ${ }^{27}$ Die Villa ist bis heute fast völlig unterirdisch

27 Zur viel diskutierten Frage des Villenbesitzers siehe etwa Mario Capasso, „Who Lived in the Villa of the Papyri at Herculaneum - A Settled Question?,“ in Zarmakoupi (Hg.), The Villa (wie Anm. 5), (89-114) und Longo Auricchio et al., La villa (wie Anm. 5), (181-192). Lucius Calpurnius Piso Caesoninus muss aufgrund seiner Verbindung mit Kampanien und Philodem, dessen Bibliothek in der Villa gefunden wurde, als wahrscheinlichster Kandidat gelten. Jedoch überrascht das Fehlen von inschriftlichen Zeugnissen zur gens Calpurnia in Herkulaneum (kann aber erklärt werden), so dass etwa Mommsen an Comparettis Identifikation des Besitzers mit Piso zweifelte (Theodor Mommsen, „Inschriftbüsten,“ Archäolog. Zeitung 38 [1880]: [32-36]). In der Folge wurden diverse andere Namen in die Diskussion eingebracht, aber keiner mit wirklich überzeugenderen Argumenten als für Piso. Darüber hinaus besteht die Möglichkeit, dass die Villa den Besitzer bzw. die Besitzerfamilie wechselte. 
gelegen und die meisten Teile sind nicht mehr zugänglich. Ihre unterirdische Erschließung durch enge Tunnel und insbesondere den Lageplan samt Verzeichnis der Fundstellen, welcher als Urdokument der Archäologie angesehen werden darf, verdanken wir dem Schweizer Ingenieur Karl Weber, der ab 1749 Alcubierre zur Seite stand und faktisch die Ausgrabungen leitete. Die zahlreichen, meist sehr gut erhaltenen Statuen und Büsten dieser Villa, die von 1750 bis 1761 (dann nochmals 1764-1765) gefunden wurden, zeugen vom immensen Reichtum des Villenbesitzers und einer beeindruckenden Prachtentfaltung. Besonders der rechteckige, überdimensionierte Innenhof der Villa (rechteckiges Peristyl) ist zu nennen. ${ }^{28}$ Auch das Belvedere mit dem eleganten Marmorfußboden ist beachtlich. Einen Nachbau der Villa anhand von Webers Ausgrabungsplan hat in den 1970er Jahren der exzentrische Milliardär Jean Paul Getty in Malibu (Kalifornien) errichten lassen, die sogenannte Getty-Villa. Zur Zeit der Erbauung der Getty-Villa lag die echte Villa in Herkulaneum noch völlig unterirdisch, ja selbst ihre Lokalisierung war unscharf, so dass man für die kalifornische Kopie bzw. Interpretation nur auf Webers Plan zurückgreifen konnte. ${ }^{29}$ Winckelmann sah die Arbeitsweise Alcubierres kritisch, ${ }^{30}$ und auch Goethe klagte später: „Jammerschade, dass die Ausgrabungen nicht durch deutsche Bergleute recht planmäßig geschehen; denn gewiss ist bei einem zufällig räuberischem Nachwühlen manches edle Altertum vergeudet worden.“31

28 Zur Villa und neuen archäologischen Ergebnissen siehe Zarmakoupi (Hg.), The Villa (wie Anm. 5).

29 Zur Getty-Villa siehe Kenneth Lapatin, „Introduction“, in Lapatin (Hg.), Buried by Vesuvius (wie Anm. 5), (1-4).

30 Winckelmann, Sendschreiben (wie Anm. 24), 19: „Dieser Mann, welcher mit den Altertümern so wenig zu tun gehabt hatte, als der Mond mit den Krebsen, nach dem Welschen Sprichworte, war durch seine Unerfahrenheit Schuld an vielem Schaden und an dem Verluste vieler schöner Sachen.“

31 Johann Wolfgang von Goethe, Italienische Reise, 2. Teil, Neapel 18. März 1787. Die Aussage erhält eine gewisse Bedeutung durch den Umstand, dass Goethe als Minister in Weimar auch für den Bergbau zuständig war und somit wusste, wovon er sprach. 


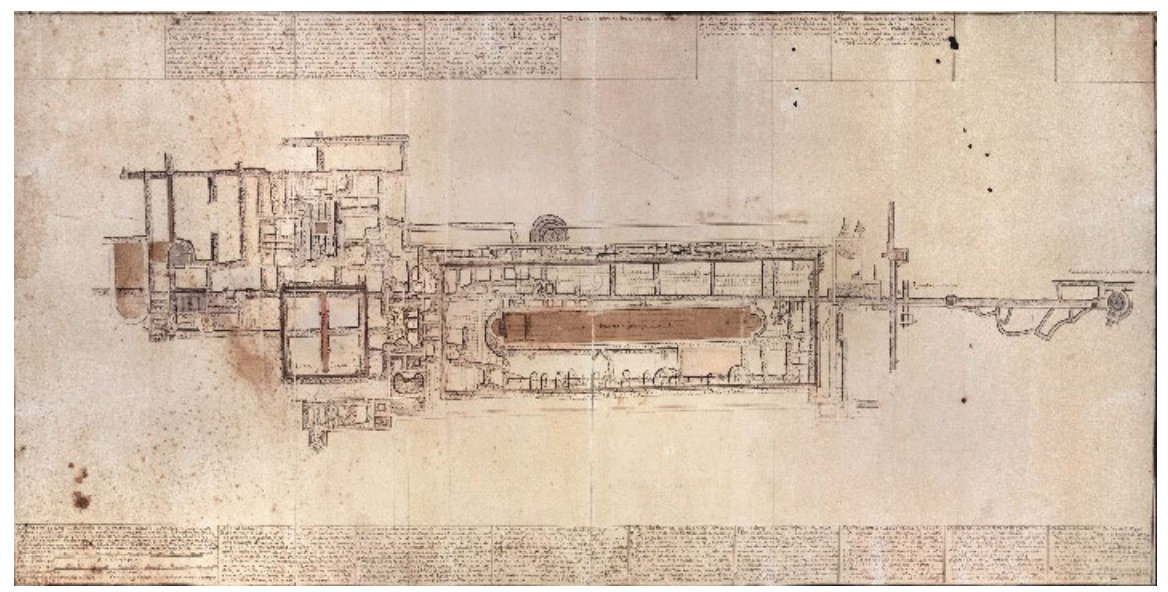

Abb. 10: Karl Webers Plan der Villa (1758)

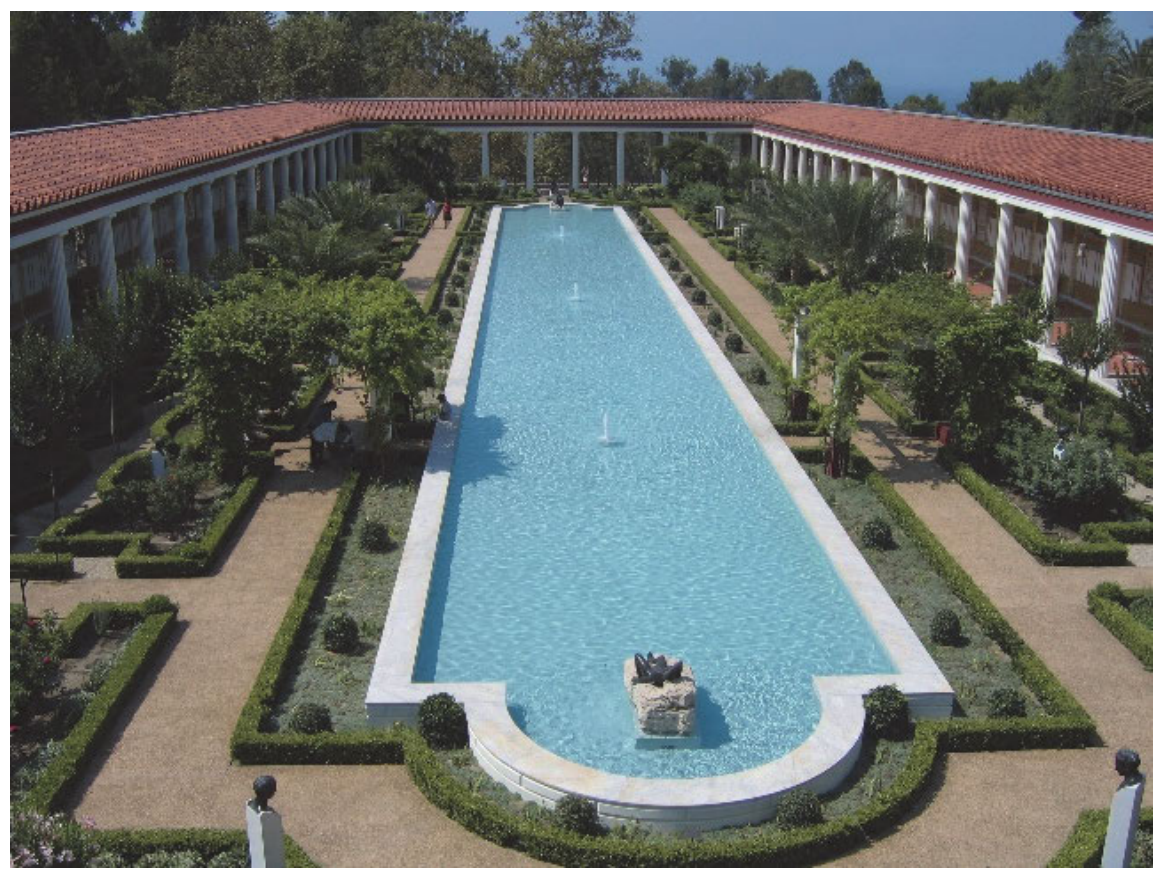

Abb. 11: Teil der Getty Villa in Los Angeles/Malibu 


\subsection{Die Entdeckung der Herkulanischen Rollen als Urmoment der Papyrologie}

Auch im Jahre 1752 scheint „manch edles Altertum vergeudet“ worden zu sein, als die unbedarften Arbeiter bei ihren Grabungen auf den vielleicht wertvollsten Schatz der Villa stießen und anfangs verkannten, worum es sich handelte. Sie fanden Gebilde, welche sie für verkohlte Wurzeln oder Fischernetze hielten und wieder wegwarfen oder zerstießen. ${ }^{32}$ Zufällig fiel eines der Objekte herunter, zerbrach und Schriftzeichen wurden sichtbar. Der Museumsleiter in Portici Camillo Paderni erkannte nun, dass es sich hier um antike, karbonisierte Schriftrollen handeln musste und reagierte prompt ${ }^{33}$ - man malt sich lieber nicht aus, wie viele Rollen zuvor durch die Unkenntnis der Arbeiter zerstört worden sind. In den nächsten Monaten fand man an weiteren Stellen des Komplexes Papyri, aber erst zwischen Februar und August 1754 kam das Gros der Papyri in einem kleinen Raum (,stanza V“ auf Webers Plan) zum Vorschein bis zu 1000 Rollen, die in Regalen an der Wand lagerten, teils auch in Schränken. ${ }^{34}$ Dieses Zimmerchen diente offenbar als Depotraum (nur für Philodems Bibliothek?), während im lichtdurchfluteten Tablinium die Papyri bevorzugt gelesen worden sein dürften. Vielleicht hatte man versucht, einige Rollen vor der Hitze und dem Ascheregen während des Vesuvausbruchs in Sicherheit zu bringen, oder sie waren aus anderen Gründen in diversen Räumen der Villa gelagert. Mit der Zeit wurde den Verantwortlichen klar, dass man hier Überreste der einzig erhaltenen Bibliothek der Antike vor sich hatte. Die Kunde von diesem Fund verbreitete sich wie ein Lauffeuer im gebildeten Europa seiner Zeit und die Rollen wurden bald als den Statuen gleichwertige Schätze des Königs angesehen.

32 Vgl. Winckelmann, Sendschreiben (wie Anm. 24), 63.

33 Die früheste Mitteilung über den Fund stammt vom 18. November 1752 (Camillo Paderni an Mead), vgl. Carlo Knight, „Le lettere di Camillo Paderni alla Royal Society di Londra sulle scoperte di Ercolano (1739-1758), “ Rendiconti dell Accademia di Archeologia Lettere e Belle Arti di Napoli 66 (1997): (13-58), 22-23. Am 19.10.1752 wurden die ersten Papyri gefunden. Überblick bei Sider, The Library (wie Anm. 5), 22-24.

34 Vgl. Mario Capasso, „Custodia e lettura dei testi nella villa ercolanese dei papiri: alcune considerazioni,“ CErc 50 (2020): (7-14), 8. 

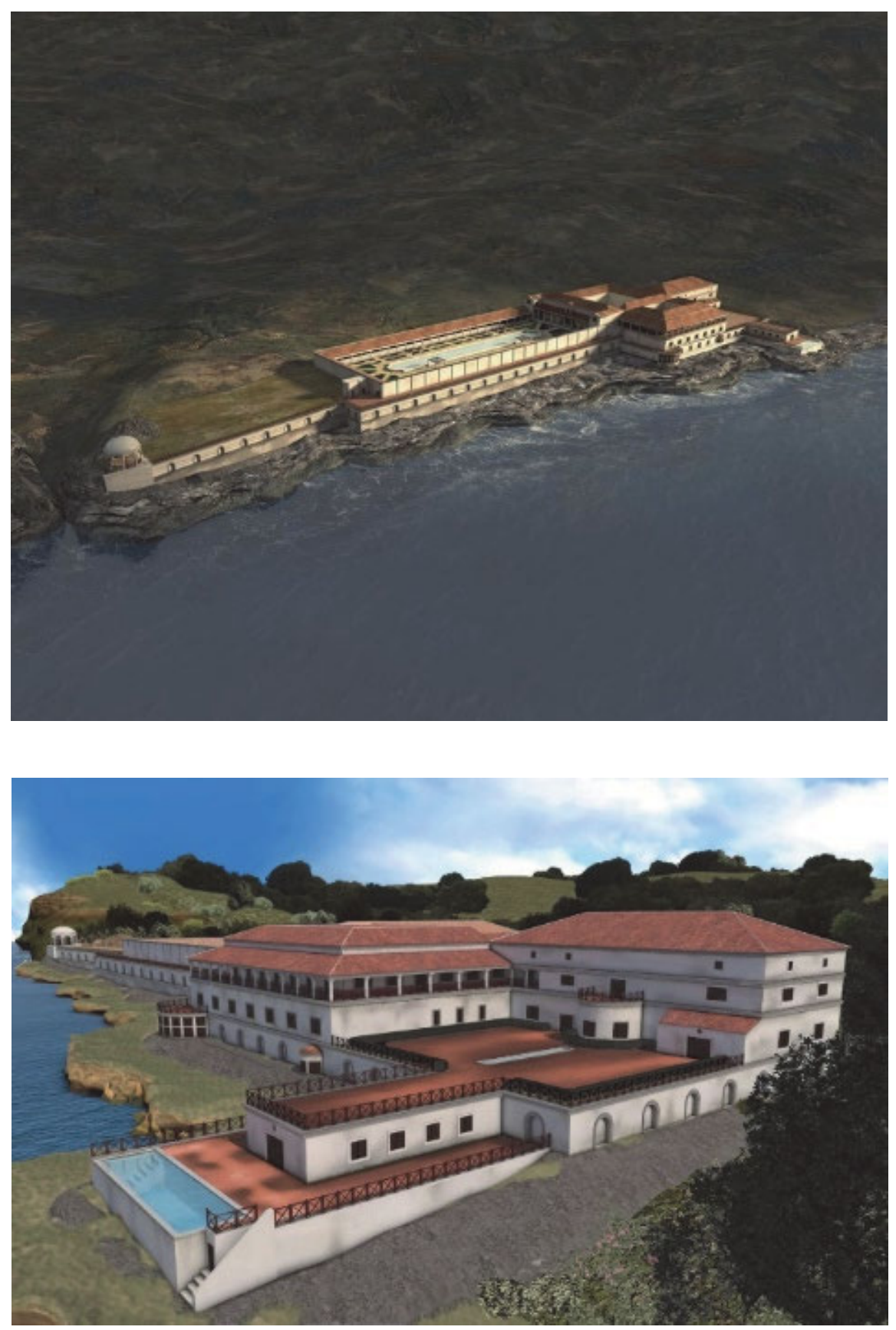

Abb. 12 und 13: Virtuelles Modell der Villa dei papiri 